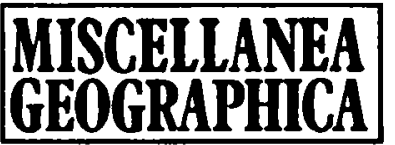

WARSZAWA 1996 Vol.7

Izabela Miszczyńska, Andrzej Walewski

\title{
LES CONSÉQUENCES D'UNE ÉVENTUELLE ÉLÉVATION DU NIVEAU DE LA MER (LE CAS DES PAYS-BAS)
}

Le phénomène de l'effet de serre avec ses diverses conséquences devient, ces derniers temps, un objet de discussions des plus fréquents, tant dans les mass média que, surtout, chez les représentants de différentes disciplines de la science. Les mass média régalent leurs destinataires avec des images, par trop simplifiées, des suites d'un réchauffement du climat qui nous menaceraient dans l'avenir immédiat. Parmi les cataclysmes évoqués le plus fréquemment, il y a une élévation du niveau des mers et une submersion de vastes étendues de terre ferme qui s'ensuivrait. Sans tenir compte de la vitesse réelle de l'évolution de ce processus, les mass média présentent souvent comme un avenir assez proche une fonte de la calotte glaciaire de l'Antarctique, une élévation du niveau des mers allant jusqu'à plusieurs dizaines de mètres, enfin une submersion menaçant des pays entiers. Des visions aussi spectaculaires d'un cataclysme global planant sur le monde sont, peut-être, utiles car elles alertent et sensibilisent les communautés humaines au problème des transformations anthropogéniques de l'environnement naturel. Mais, en ce qui concerne les chercheurs, leur rôle consiste à analyser les phénomènes qui se manifestent et à mettre au point un pronostic solidement fondé. A ce propos, nous pouvons citer un pronostic tout récent concernant les transformations du rendement de maiis dans les pays de la Communauté Européenne qui seraient la conséquence d'éventuels changements du climat. Ce pronostic a été publié par J.Wolf et C.A. van Diepen dans le ${ }^{\circ} 29$ de la revue Climatic Change de 1995.

Partant du cas des Pays-Bas, nous allons tenter de déterminer les conséquences qu'aurait une élévation probable du niveau des océans pour l'aménagement de ce pays.

Le choix des Pays-Bas a été dicté par le caractère particulier de cet État - littoral, situé en partie sur les plaines et en partie dans la zone de dépression - et par là-même spécialement exposé en cas d'une élévation éventuelle du niveau de la mer. De plus, c'est un pays intensivement mis en valeur.

Le premier problème à résoudre est la perspective de temps concernant les recherches. Le phénomène de l'élévation du niveau des mers (attribué à 


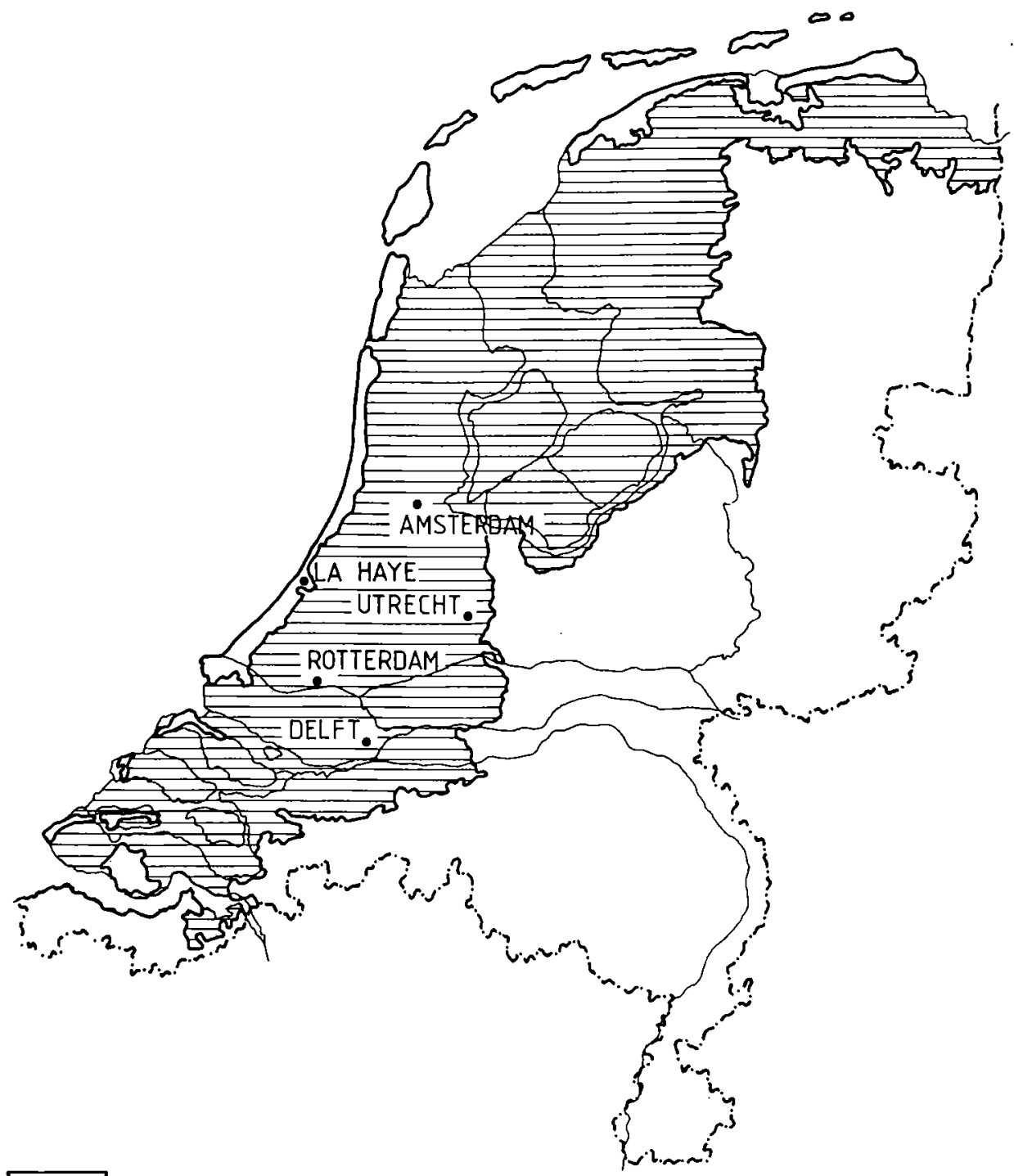

terres menacées de submersion

Fig. 1. Le territoire des Pays-Bas après l'élévation du niveau de la mer de $100 \mathrm{~cm}$

l'effet de serre qui ne cesse de croître) se développe à une allure assez lente si on le compare avec la durée de la vie de l'homme. La plupart de chercheurs avancent, partant des calculs effectués, que le niveau des mers s'est accru de 10-15 cm durant la dernière centaine d'années. Une telle vitesse de ce processus incitait à admettre une perspective suffisamment éloignée pour qu'un changement du niveau des mers soit assez important. Finale- 
ment, on a décidé d'adopter l'analyse d'une situation qui semble la plus probable dans cent ans c'est-à-dire vers la fin du XXI $\mathbf{s}$. Parmi les facteurs ayant décidé de l'adoption de cette date il y a le fait que de nombreux pronostics concernent cette époque particulière.

Les pronostics de l'importance de l'élévation du niveau des mers pour l'année 2100 , mis au point par de nombreux auteurs, different les uns des autres de façon considérable. La cause principale en est la différence entre les pronostics concernant la montée de la température moyenne sur la Terre - différents auteurs admettent différentes grandeurs de l'émission de $\mathrm{CO}_{2}$ dans l'atmosphère à cette époque. Ainsi, ce n'est point étonnant que les différences entre des pronostics concernant l'élévation du niveau des mers jusqu'à l'an 2100 soient si grandes et vont depuis quelques centimètres jusqu'à $3,5 \mathrm{~m}$. Les grandeurs les plus fréquentes oscillent de 60 à $120 \mathrm{~cm}$. L'analyse dont il a été question plus haut devait être basée sur la grandeur de $100 \mathrm{~cm}$, acceptée par de nombreux auteurs. Néanmoins, il faut se rendre compte que la valeur de pronostics concernant un phénomène tellement complexe et un avenir si éloigné doit être, par la force des choses, limitée. Un pronostic concernant l'état d'aménagement d'un pays dans cent ans serait encore plus risqué et relèverait plutôt de la science-fiction que de l'analyse scientifique.

Les Pays-Bas sont un pays qui, depuis des siècles, risque d'être submergé par les eaux de mer. Les Néerlandais ont non seulement réussi à protéger leur pays contre les submersions, mais sont également parvenus à conquérir sur mer des terres nouvelles (plusieurs milliers d'hectares) et les aménager. Les protections qui existent de nos jours sont effectives par rapport au niveau actuel de la mer mais peuvent être insuffisantes si le niveau de la mer s'élève de $100 \mathrm{~cm}$. Une analyse cartographique a démontré que dans ce cas plus de 16 mille $\mathrm{km}^{2}$ de terres (44\% de la superficie du pays) seraient submergés. Les plus exposés sont les terrains situés dans le voisinage immédiat de la mer. Ce sont, pour la plupart, les terres asséchées, auparavant submergées par la mer (les polders). Certaines provinces seraient submergées complètement (Flevoland), d'autres en grande partie (Hollande-Méridionale - $91 \%$, Hollande-Septentrionale $-88 \%$, Zélande $-82 \%$, Frise $-81 \%$, Utrecht $-62 \%$ ). Les terres menacées de submersion comptent parmi les plus fortement peuplées, avec les plus grandes villes du pays: Amsterdam, Rotterdam, Utrecht, Haarlem. Ce sont des villes à un patrimoine culturel important avec, entre autres, des édifices anciens de valeur unique dont la submersion serait une perte difficile à estimer. 70 villes au total $(30 \%)$ seraient submergées. Dans les terrains menacés de submersion se trouvent des centres industriels d'une grande importance dont la perte ferait diminuer le potentiel industriel du pays de $43 \%$. Il s'agit surtout de l'industrie chimique et les industries métallurgiques. La submersion des plaines basses équivaudrait à la perte des terres cultivables ayant la plus grande valeur agricole. Ainsi, l'eau de mer submergerait $50 \%$ de la surface 
agricole utile (SAU), $40 \%$ de sols arables où l'on pratique des cultures intensives hautement spécialisées, enfin $53 \%$ de prés et de pâturages.

Les résultats de l'analyse cartographique présentés plus haut permettent d'évaluer l'importance des pertes des Pays-Bas qui seraient la conséquence d'une élévation du niveau de la mer de $100 \mathrm{~cm}$. Mais, puisque ce processus aurait été plutôt lent, il serait possible d'envisager la mise en place de dispositifs de protection qui permettraient d'éviter la réalisation de ces scénarios catastrophiques. Les travaux nécessaires sont pourtant onéreux et, ce qui plus est, ils ne résoudraient pas tous les problèmes. Il faudrait s'attendre, par exemple, à un renforcement de certains processus nocifs dont, entre autres, l'intrusion des eaux salées dans les lits de fleuves, la salinisation des sols et la destruction d'une partie de réservoirs naturels d'eau potable.

En conclusion, nous aimerions souligner que l'analyse effectuée se rapporte à une situation hautement probable c'est-à-dire une élévation du niveau des mers de $100 \mathrm{~cm}$ dans cent ans. Cette analyse montre, sur l'exemple d'un seul pays, l'importance des conséquences d'un changement de l'environnement pour le fonctionnement de l'homme. Évidemment, ce ne soní que spéculations basées sur des hypothèses adoptées concernant les changements du climat. Il faut pourtant se rendre compte que le degré de fiabilité demeure controversé et prête toujours à discussion. 